\title{
One-Stop-Test solutions for autonomous driving
}

Frank Heidemann

SET GmbH

This manuscript is not available according to publishing restriction.

Thank you for your understanding. 